\section{Synthesis of (+)-Cyclobutastellettolide B}<smiles>C#CCCCC1OC1CCCCC</smiles>

A

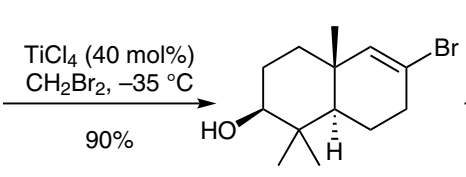

1. $t$-BuLi, $\mathrm{Et}_{2} \mathrm{O}-$ hexanes $(5.7: 1),-78^{\circ} \mathrm{C}$

then DMF, $-78^{\circ} \mathrm{C}$ to r.t.

2. $\mathrm{NaBH}_{4}, \mathrm{THF}-\mathrm{MeOH}(10: 1), 0{ }^{\circ} \mathrm{C}$

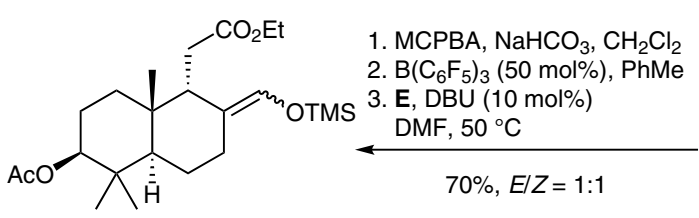

$\mathbf{F}$ $\mathrm{CH}_{2} \mathrm{Cl}_{2}$-hexanes $(1.7: 1), 0^{\circ} \mathrm{C}$ then $\mathrm{CH}_{2} \mathrm{l}_{2}, \mathrm{CH}_{2} \mathrm{Cl}_{2}, 0^{\circ} \mathrm{C}$ to r.t.

$88 \%, \mathrm{dr}=1.1: 1$

Simmons-Smith cyclopropanation

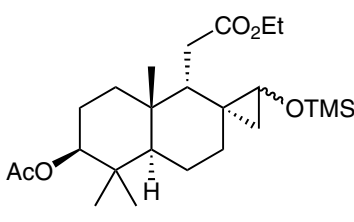

G<smiles>CC1(C)[C@H](O)CC[C@H]2CC(CO)=C[C@]21C</smiles>

C<smiles>FC(=N[AsH3])O[Mg]</smiles><smiles>C=C1CC[C@H]2C(C)(C)[C@@H](OC(C)=O)CC[C@]2(C)[C@H]1CC(=O)OCC</smiles>

D

1. $\mathrm{PvOH}(5 \mathrm{~mol} \%)$ $\mathrm{CH}_{3} \mathrm{C}(\mathrm{OEt})_{3}, \Delta$

2. $\mathrm{AcOH}-\mathrm{Ac}_{2} \mathrm{O}(1: 1)$ then $\mathrm{Et}_{3} \mathrm{~N}$ DMAP (50 mol\%) $0^{\circ} \mathrm{C}$ to r.t. $84 \%$ Johnson-Claisen rearrangement
Category

Synthesis of Natural

Products and

Potential Drugs

\section{Key words}

(+)-cyclobutastellettolide B

Johnson-Claisen rearrangement

Simmons-Smith cyclopropanation

Norrish-Yang cyclization

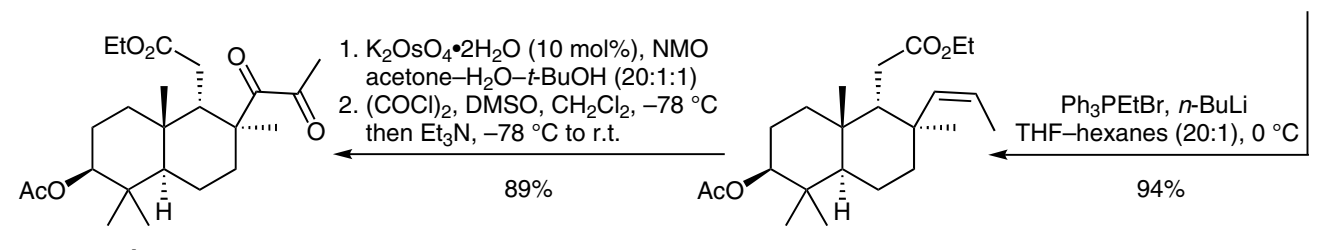

J<smiles>CC(=O)O[C@H]1CC[C@]2(C)[C@H](C)[C@@](C)(C=O)CC[C@]2(C)C1(C)C</smiles>
H

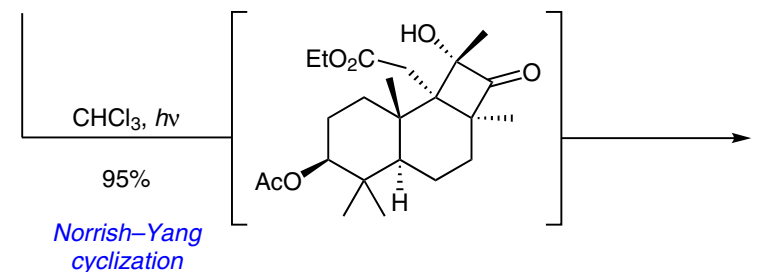<smiles>CC(=O)O[C@H]1CC[C@@]2(C)[C@@H](CC[C@]3(C)C(=O)[C@]4(C)OC(=O)C[C@@]324)C1(C)C</smiles>

(+)-Cyclobutastellettolide B

Significance: (+)-Cyclobutastellettolide $B$ is a natural product isolated in 2019 from a Stelleta sp. sponge (S. A. Kolesnikova et al. J. Nat. Prod. 2019, $82,3196)$. It features a 6/6/4 fused tricyclic core, including a fully substituted cyclobutane, rendering it an interesting target for total synthesis.
Comment: Vinyl bromide B was rapidly elaborated into ester $\mathbf{D}$ using a Johnson-Claisen rearrangement. Installation of the $\alpha$-tertiary aldehyde in $\mathbf{H}$ was achieved via ring opening of the cyclopropane in G. Wittig reaction followed by oxidation yielded diketone J which smoothly underwent the desired Norrish-Yang cyclization, followed by lactonization, to yield the natural product. 